\title{
Cancers et autres maladies non transmissibles : vers une approche intégrée de santé publique
}

\section{Cancer and other non-communicable diseases: to an integrated public health approach}

\author{
A. Ly \\ C) Springer-Verlag France 2012
}

Les maladies non transmissibles (MNT) font une percée épidémiologique partout dans le monde [1]. On ne peut rester impavide ni inactif devant une telle situation.

Si les pays développés ont été affectés depuis très longtemps, les pays en développement (PED) ont désormais une incidence de ces maladies en progression constante. Cette nouvelle donne épidémiologique montre bien que les MNT ont tendance à s'installer durablement dans les PED et que le phénomène n'est ni transitoire ni cantonné d'ailleurs à la seule classe sociale des plus favorisés au sein de ces pays [2-5]. Les pays du Sud et les pays à revenus intermédiaires font face à un double fardeau que constituent les MNT et les maladies transmissibles (MT).

Ces transformations épidémiologiques battent en brèche nos paradigmes, rendent obsolètes nos grilles d'analyses et modifient en profondeur les frontières d'antan entre et à l'intérieur de nos sociétés.

La conférence de haut niveau sur ces maladies qui s'est tenue à New York (États -Unis) les 19 et 20 septembre 2012 sous l'égide de l'assemblée générale des Nations Unies a permis d'ouvrir le débat et d'offrir une tribune internationale à des $\mathrm{ONG}$, des représentants de gouvernements de divers pays du monde, universitaires et professionnels de santé, de l'environnement, de l'agroalimentaire, de l'urbanisme, du développement durable, de l'économie solidaire [3]. Il était temps. Les MNT ne figurent que rarement à l'agenda de la politique internationale. D'ailleurs, elles ne font pas partie des objectifs du millénaire pour le développement $[6,7]$. Et pourtant, elles représentent de loin les premières causes de mortalité dans le monde soit 36 millions de décès par an [1].

La diversité des participants à ce sommet de l'ONU sur les MNT est à la mesure de la complexité du problème. La compréhension et la recherche de solutions à ces nouveaux enjeux de santé publique n'incombent plus aux seuls scientifiques et professionnels de santé.

\footnotetext{
A. Ly $(\bowtie)$

Afrocancer, BP 60751, F-75827 Paris cedex 17, France

e-mail : adamaly@gmail.com
}

De même, les dimensions économiques, sociales et environnementales ont été soulignées et sont incontournables dans la recherche de solutions. Elles constituent, ensemble, le carburant qui alimente la dynamique d'évolution des MNT. Faisant partie du problème, elles doivent être des éléments caractéristiques de la prise en charge globale des MNT.

Enfin, que doit-on mettre sous le terme « maladies non transmissibles " ? Une définition officielle peut-elle être adoptée ? Les utilisations sont larges et les frontières souvent floues : maladies cardiovasculaires, cancers, diabètes, maladies respiratoires, santé mentale, allergies, maladies autoimmunes, maladies neurodégénératives,...

Ces maladies sont aussi appelées maladies chroniques parce que leurs prises en charge se font à long terme ou durant toute la vie dès lors qu'elles sont installées.

De la même manière, des MNT sont nommées parfois maladies émergentes parce qu'elles ont des indicateurs épidémiologiques (taux d'incidence) qui sont en très nette augmentation au cours d'un certain temps dans un espace géographique donné. Deux qualificatifs qui ne sont pas spécifiques aux MNT. Des MT peuvent être émergentes et chroniques comme le sida est en passe de le devenir dans les pays du Nord. Par ailleurs, c'est un truisme de dire que tout ce qui n'est pas « transmissible » est « non transmissible ». Une définition par défaut manquerait de clarté, de concision et diluerait la dimension pédagogique recherchée.

Un bon niveau de pertinence pourrait être une classification en fonction des facteurs de risques communs à des MNT en dépit de mécanismes physiopathologiques différents. Par exemple, le tabagisme, l'alcoolisme, une alimentation non équilibrée, l'absence d'activité physique, le surpoids (ou l'obésité) sont des facteurs de risques communs aux cancers, aux maladies cardiovasculaires, au diabète de type 2 et aux maladies respiratoires. À ces quatre groupes de pathologies, on attribue $63 \%$ de la mortalité mondiale et $80 \%$ des décès dans les pays en développement et les pays à revenus intermédiaires $[1,3,8,9]$ (Fig. 1).

D'un point de vue pratique et financier, des actions de prévention communes peuvent être élaborées. En effet, 


\section{Répartition de la mortalité par MNT (\%)}

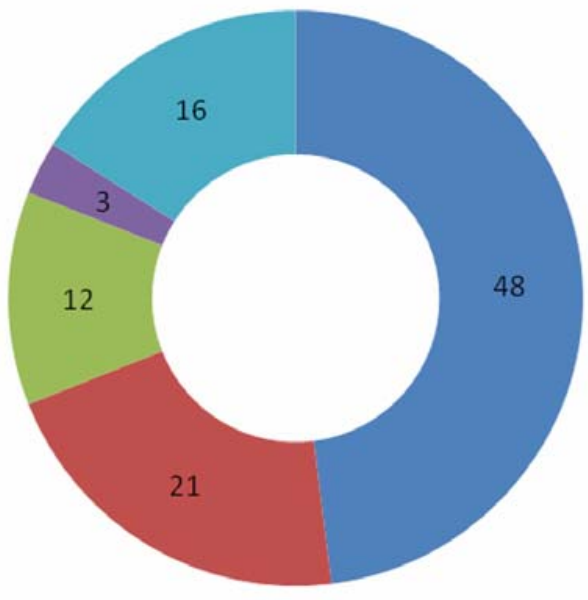

Maladies cardiovasculaires

- Cancers

Maladies respiratoires

Diabéte type 2

Autres MNT

Fig. 1 Répartition des maladies non transmissibles (MNT) selon leur mortalité totale (36 millions de décès en 2008$)$ soit $63 \%$ de la mortalité mondiale estimée à 57 millions. Source WHO [1]

$80 \%$ des maladies cardiovasculaires, des cas de diabètes de type 2 et $1 / 3$ des cancers peuvent être évités par une meilleure hygiène de vie et des dépistages précoces $[3,8,9]$.

Ce sont de telles politiques intégrées de santé publique qui doivent être promues. L'absence d'action de prévention efficace aurait pour conséquence une hausse de la mortalité des MNT de $17 \%$ au cours des dix prochaines années. Dans ce scénario, la région africaine serait plus impactée avec $27 \%$ d'augmentation de la mortalité suivie des pays de la Méditerranée orientale (25\% d'augmentation) [1,3].

Les MNT sont d'autant plus pernicieuses qu'elles sont imputables de $29 \%$ des décès (soit environ 10 millions de morts) avant l'âge de 60 ans dont $80 \%$ dans les pays pauvres et à revenus intermédiaires ( 8 millions de décès) $[1,3,8]$. Ce sont ainsi les forces vives de la société qui meurent prématurément rendant plus pauvres les pays les plus vulnérables et réduisant à néant leurs efforts de développement, d'éducation et de formation.

En sus, les générations futures, les enfants et les jeunes adultes, sont particulièrement exposées à ces facteurs risque communs aux MNT. On estime que 43 millions d'enfants en âge préscolaire dans le monde souffrent de surpoids ou d'obésité [3]. C'est aussi, au cours des premières années de la vie, que nombre d'entre eux sont exposés au tabac et à l'alcool et en sont souvent victimes.

Finalement, c'est notre responsabilité individuelle et collective qui est questionnée comme jamais elle ne l'a été. La riposte face à cette grande menace des maladies non transmissibles incombe à tous, aux institutions, aux états, aux Organisations non gouvernementales (ONG). Ce qui doit rendre nos discussions utiles et responsables et nos polémiques stériles et inopportunes.

Aux conséquences socioéconomiques dramatiques prévues, on opposera une politique de prévention réaliste dont les axes stratégiques seront adaptés aux populations locales, à leurs vécus culturels et à leurs possibilités de participation (pouvoir d'achat, niveau de scolarisation,...).

L'adoption, à cour terme, de mesures préventives et thérapeutiques est non seulement nécessaire mais relève du champ des possibles. Selon l'OMS, avec 1,20 dollars par an et par personne, des stratégies efficaces peuvent être mise en place pour une prise en charge globale des MNT dans les pays à revenus faibles et ceux à revenus intermédiaires. Le budget annuel estimé est de 11,4 milliards de dollars [3,10-12].

En comparaison, au plan économique, les pertes de productivité (morbidité, mortalité prématurée,...) liées aux MNT s'évaluent en milliers de milliards de dollars. Au cours des 15 prochaines années, les MNT coûteront 7000 milliards de dollars à ces pays dont les économies restent fragiles [10].

Outre ces aspects sanitaires, économiques et sociétaux, les aspects législatifs et fiscaux devront compléter les dispositifs de surveillance en amont et faciliter l'accès aux outils thérapeutiques.

En effet, des réflexions devront être engagées pour taxer ou légiférer davantage sur les prix du tabac, de l'alcool, des aliments trop salés, trop gras ou trop sucrés afin de limiter l'exposition aux facteurs de risques des MNT. 
Parallèlement, l'offre thérapeutique peut être améliorée si les réglementations sur les brevets, les prix des médicaments et des produits de santé sont réexaminées à l'aune de ces enjeux cruciaux de santé par l'Organisation mondiale du commerce, l'Organisation mondiale de la santé, les firmes pharmaceutiques et les gouvernements des pays restés impuissants devant la menace des MNT.

Au total, l'impact des MNT ira grandissant au cours de ce siècle. Des stratégies renouvelées de santé publique sont d'ores et déjà à mettre en place au niveau local et global pour relever le défi. Les moyens et les possibilités de prévention, de traitements et de financements existent et sont mobilisables si les responsabilités politiques, économicofinancières et scientifiques s'allient, comme s'associent du reste les différents facteurs de risque des MNT, pour être à la bonne hauteur de ces enjeux de santé internationale.

Des efforts substantiels sont attendus du côté des gouvernements africains qui s'étaient engagés dans la déclaration d'Abuja en 2001 à augmenter la part de leur PIB investie dans la santé à $15 \%$. Depuis, force est de constater que les promesses n'ont pas été tenues et que les dépenses de santé de ces pays dont la croissance démographique est en régulière hausse n'excèdent pas $5,5 \%$ du produit intérieur brut (PIB) [13]. Cette faiblesse constante des ressources allouées à la santé par certains pays ne peut permettre de résoudre ni les énormes besoins d'investissements en structures et infrastructures ni la forte demande médicale des populations.

Les MNT sont le défi médical de ce siècle. Leur prise en charge responsable, éthique et volontariste tant au niveau socioéconomique, sanitaire que politique ne sera pas optionnelle.

\section{Références}

1. WHO (2012) World health statistics 2012 Geneva, World Health Organization

2. Mc Carthy M, Maher D, Ly A, Agbor Ako NDIP (2010) Developing the agenda for European Union collaboration on noncommunicable diseases research in Sub-Saharan Africa. Health Research Policy and Systems, 8:13 doi: 10.1186/1478-4505-8-13

3. Alwan AD, Galea G, Stuckler D (2011) Le développement en danger : s'attaquer aux maladies non transmissibles lors de la réunion de haut niveau des Nations Unies, Bulletin de l'Organisation mondiale de la Santé 89:546-546A

4. Sylla BS, Wild CP (2012) Cancer burden in Africa in 2030: Invest today and save tomorrow. J Afr Cancer 4:1-2

5. Ly A (2011) Enjeux et perspectives de la prévention des cancers dans les pays en développement J Afr Cancer 3:268-72

6. ONU (2000) Les objectifs du millénaire pour le développement. Déclaration du millénaire sommet du millénaire, New York, septembre 2000 www.un.org/french/millenniumgoal

7. Shah E, Liam S (2008) Non-communicable diseases in low and middle-income countries: a priority or a distraction? Int J Epidemiol 34:961-6

8. Global status report on non communicable diseases 2010 (2011) Description of the global burden of NCDs, their risk factors and determinants World Health Organization, Geneva, April 2011, 176 p, ISBN: 9789241564229

9. Plan d'action 2008-2013 (2010) Pour la Stratégie mondiale de lutte contre les maladies non transmissibles. Organisation mondiale de la Santé, Genève, 2010

10. WHO, World Economic Forum (2011) From burden to "best buys": Reducing the economic impact of NCDs in low and middle-income countries, Geneva, World Health Organization

11. WHO Communiqué de presse (2011) Endiguer les maladies non transmissibles à moindre coût. http://www.who.int/mediacentre/ news/releases/2011/NCDs solutions 20110918/fr/ Dernier accès le 5 juin 2012

12. WHO (2011) Scaling up action against non communicable diseases: How much will it cost? Geneva, World Health Organization

13. WHO (2011) Abuja declaration: Ten years On Geneva, World Health Organization 